\title{
HIV-1 Epidemiology, Genetic Diversity, and Primary Drug Resistance in the Tyumen Oblast, Russia
}

\author{
Natalya M. Gashnikova, ${ }^{1}$ Ekaterina M. Astakhova, ${ }^{1}$ \\ Mariya P. Gashnikova, ${ }^{1}$ Evgeniy F. Bocharov, ${ }^{1}$ Svetlana V. Petrova, ${ }^{2}$ \\ Olga A. Pun'ko, ${ }^{2}$ Alexander V. Popkov, ${ }^{2}$ and Aleksey V. Totmenin ${ }^{1}$ \\ ${ }^{1}$ State Research Center of Virology and Biotechnology Vector, Koltsovo, Novosibirsk Oblast 630559, Russia \\ ${ }^{2}$ Tyumen Regional Center for Prevention and Control of AIDS and Infectious Diseases, Ul. Novaya 2, Tyumen, Russia
}

Correspondence should be addressed to Natalya M. Gashnikova; ngash@ngs.ru

Received 3 August 2016; Revised 23 September 2016; Accepted 3 October 2016

Academic Editor: Llibre Josep-Maria

Copyright (C) 2016 Natalya M. Gashnikova et al. This is an open access article distributed under the Creative Commons Attribution License, which permits unrestricted use, distribution, and reproduction in any medium, provided the original work is properly cited.

\begin{abstract}
Introduction. Specific molecular epidemic features of HIV infection in Tyumen Oblast (TO), Russia, were studied. Methods. The genome sequences encoding HIV-1 protease-reverse transcriptase, integrase, and major envelope protein were examined for 72 HIV-1 specimens isolated from the TO resident infected in 2000-2015. Results. The recorded prevalence of HIV-1 subtype A (A1) is 93.1\%; HIV-1 subtype B continues to circulate in MSM risk group (1.4\%). Solitary instances of HIV-1 recombinant forms, CRF63_02A1 (1.4\%) and CRF03_AB (1.4\%), were detected as well as two cases of HIV-1 URF63_A1 (2.8\%). Phylogenetic analysis showed no HIV-1 clustering according to the duration of infection and risk groups but revealed different epidemic networks confirming that HIV infection spread within local epidemic foci. A high incidence of CXCR4-tropic HIV-1 variants and a higher rate of secondary mutations influencing the virus fitness (K20R, L10V, and I) are observed among the virus specimens isolated from newly infected individuals. Conclusions. The current HIV-1 epidemic in TO develops within the local epidemic networks. Similar to the previous period, HIV-1 subtype A is predominant in TO with sporadic cases of importation of HIV-1 recombinant forms circulating in adjacent areas.
\end{abstract}

\section{Introduction}

The Russian Federation is now in the list of regions with a high rate of HIV epidemic spread [1]. In 2016, the total number of officially registered persons with diagnosed HIV infection in Russia exceeds one million. The HIV prevalence among Russian citizens in 2015 continued to grow. A high HIV prevalence (over $0.5 \%$ of total population) is observed in 26 regions, housing $41.5 \%$ of the population of this country [2].

The cases of first mass detection of HIV-1 infection in the territories of Russia and other newly independent states of the former Soviet Union date back to 1996. In 2000-2001, local dramatic outbreaks of HIV-1 infection were recorded in many regions of Russia; these outbreaks stemmed from an active spread of HIV-1 among injection drug users (IDUs). HIV-1 subtype A was included into the risk group for IDUs and continued its spread in many territories of this country [3-8]. The Tyumen Oblast (TO) is a federal subject of Russia with the administrative center in the city of Tyumen (Figure 1). This territory with an area of $1435200 \mathrm{~km}^{2}$ has 3615485 residents, with the urban population accounting for $80.12 \%$. TO was among the first Russian territories actively involved in the HIV epidemic: 767 individuals diagnosed with HIV infection were at once recorded there in 1999 and 1067 individuals in 2000. A drastic increase in the HIV prevalence rate in TO in 1999-2002 was associated with a spread of opioid drugs (fresh opium and heroin), so that the infection cases associated with drug abuse accounted for $98-78 \%(98.4 \%$ in $1999,96.4 \%$ in $2000,93.7 \%$ in 2001 , and $78.4 \%$ in 2002). The maximal number of cases was recorded in the city of Tyumen and neighboring areas: the share of HIV-infected individuals living in the city of Tyumen in 2000 amounted to $73.6 \%$ of the total detected cases. 


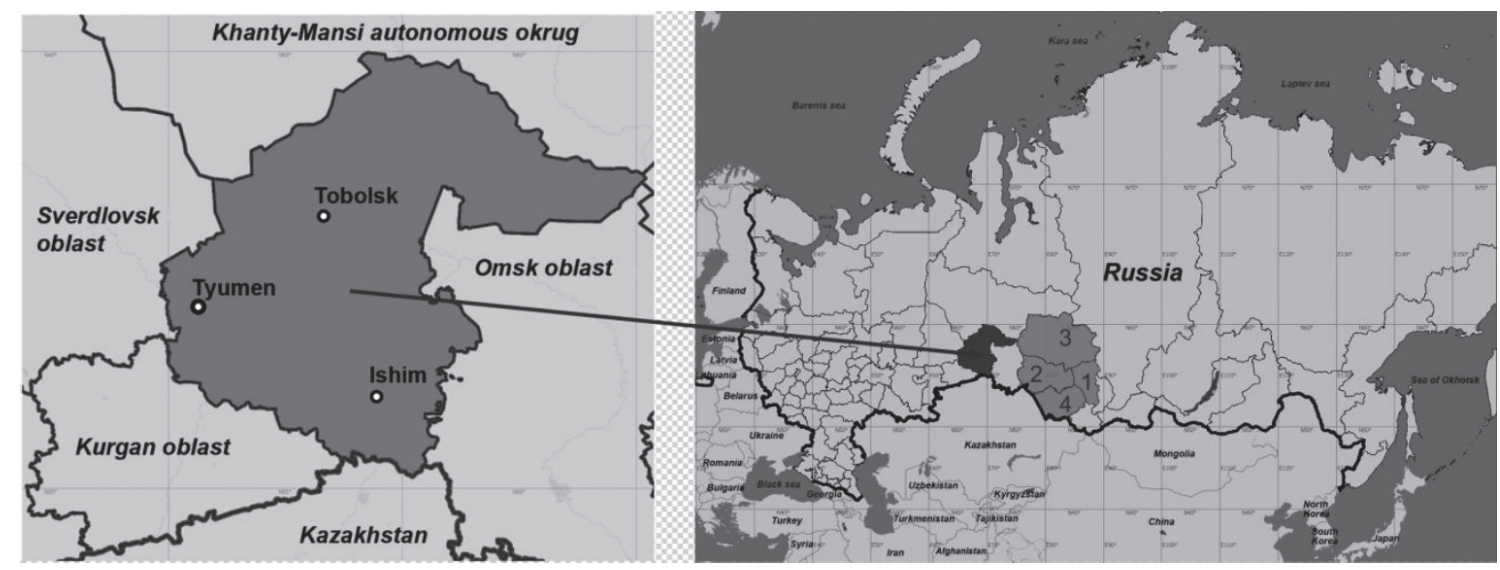

FIGURE 1: Geographical location of the Tyumen Oblast (highlighted in dark gray) and neighboring territories, Russia. (1) Kemerovo Oblast; (2) Novosibirsk Oblast; (3) Tomsk Oblast; and (4) Altai Krai.

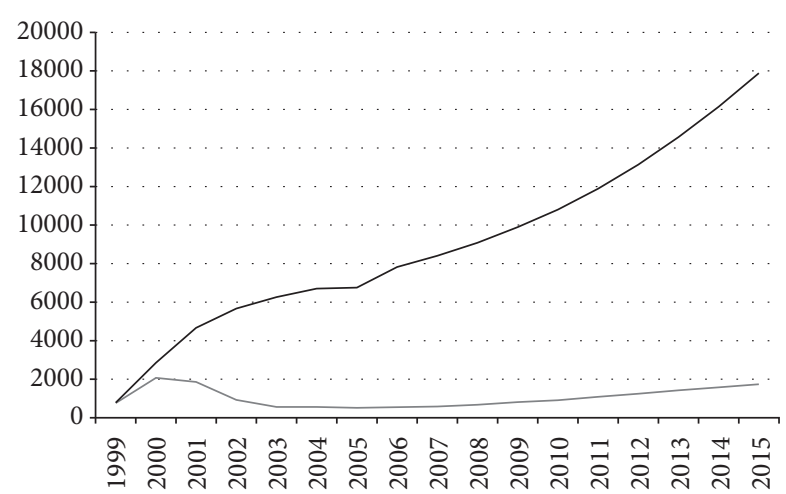

FIgUre 2: Dynamics of the HIV-1 epidemic in the Tyumen Oblast. Gray line denotes the newly diagnosed infection cases and black line total number of HIV-infected individuals.

Since the very beginning of mass HIV spread in TO, several different programs were implemented there that focused on HIV prevention and treatment (information and education of various population cohorts and organization of laboratory facilities allowing for timely detection, prescription, and efficiently control of the relevant therapies) and on the control of drug abuse. This allowed for coping with the drastic growth in the epidemic: its rate in 2003-2008 considerably slowed down (Figure 2). During this period, the minimal growth in HIV morbidity was observed (below $2 \%$ or even negative); however, the share of women among the newly diagnosed HIV infection cases increased as well as the contribution of sexual transmission (50\% in 2004), while the share of teenagers among HIV-infected individuals reduced. During the overall epidemic, sexual transmission was to a larger degree characteristic of women: up to $70 \%$ are annually infected via heterosexual contacts and only $22-27 \%$ via drug versus $73-78 \%$ of men infected when using drugs and only $29-33 \%$, via sexual contacts.

Since 2008, numerous new drugs and homemade drug variants have appeared in the drug market. Emergence of new psychoactive substances offered as a "legal" alternative for the known drugs [9] controlled at an international level had a significant effect on the further development of HIV epidemic in many regions of this country. The new psychoactive substances (the so-called "synthetics" and "salts") are marketed under harmless names as "salts," such as, bath salts, legal highs, speed euphoria, Barnyi, Cico, Cristalius SEX, Cristalius LOVE, Miff, Ivory Wave, Liquid Snow Berry, Snow Blow, and Spice. These substances were sold almost legally and were considerably less expensive as compared with classical drugs, making them easily accessible.

Synthetic psychoactive substances differ from the opioid drugs not only by their price and accessibility. These substances have a lower toxic threshold, which decreases the fear of death caused by overdosing, and have no effect of withdrawal; that is, they are used not to eliminate the withdrawal symptoms, but to reach an altered state of consciousness (euphoria or almightiness). Specific features of synthetics consist not only in their effect on the nervous system (disturbance of locomotion, memory, and sensitivity to pain as well as an increase in sexual sensations), but also in the injury of the immune system. The dependence on synthetics develops very rapidly. Ever new synthetics with changed structures and different administration methods (injection, smoking mixtures, etc.) constantly appear on the marker with addition of such substances to the lists of illegal drugs [10].

Emergence of such psychoactive substances involved many new persons into practicing risk behavior. The individuals intravenously injecting synthetics lose control over their behavior and start to share syringes. The practice of mindless unprotected sex becomes common when using some synthetics, including smoking mixtures. Consequently, the number of newly diagnosed HIV cases started to increase in TO in the second half of 2009. The epidemic history of $\mathrm{HIV}^{+} \mathrm{TO}$ residents ever more frequently contains the use of synthetic psychostimulants.

Characteristic of the subsequent years is a more active aggressive spread of psychoactive substances in many Russian regions. It is possible to purchase synthetics just calling by phone or via the internet. The situation continued to deteriorate because of an increased accessibility of these 
synthetic psychostimulants and spread of the drugs effective in sexual disinhibition; in addition, many drug addicts commenced practicing combined (multiple) drug use. For 20112014, men were prevalent among the newly detected $\mathrm{HIV}^{+}$ individuals, also suggesting activation of the drug-associated HIV infection route. Nonetheless, a stable decrease in the annual rate of HIV morbidity growth has been observed starting from 2011 on the background of an absolute increase in the HIV incidence.

As of May 01, 2016, 18423 cases of HIV infection were recorded in TO. According to the HIV prevalence, TO is the eighth in the list of Russian regions (1107.0 $\mathrm{HIV}^{+}$individuals per 100000 population) and thirteenth according to the morbidity rate (106.6 per 100000 population).

Active HIV-1 outbreaks in the regions adjacent to TO (Novosibirsk, Tomsk, and Kemerovo Oblasts; Figure 1) recorded in 2008-2014 were associated with a drastic change in genetic characteristics of the circulating viruses, namely, replacement of the earlier circulating HIV-1 subtype A by CRF63_02A1 [11-13]. The studies of the HIV-1 variants circulating in TO have not been conducted so far. A high HIV-1 prevalence in TO and a long history of antiretroviral therapy (ART) there justify the interest to assess the specific molecular genetic features of the virus population circulating there and analyze HIV-1 primary resistance.

\section{Materials and Methods}

In total, 74 TO residents with diagnosed HIV infection were involved in this study. Peripheral blood of the HIVinfected individuals was sampled by medical staff of the Tyumen Regional Center for the Prevention and Control of AIDS and Infectious Diseases from September to December 2015 with pretest and posttest psychological consultations; epidemiologists obtained the written informed content from the participants and questioned them. The study of clinical blood samples complied with the ethical standards of the Helsinki Declaration of 1975 (http://www.wma.net/en/ 30publications/10policies/b3/), revised in 2008. The study projects were approved by the local ethical committee (protocols nos. 7 and 15 of March 20, 2013, and March 4, 2015). The blood samples were linked with demographic and clinical data via coded ID numbers according to the requirements of medical ethics in Russia. All participants were ART-naïve before the tests. The recorded characteristics for patients included their gender, age, transmission route, estimated time of infection, dates of the last negative and first positive tests for HIV, drug use, viral load, and CD4 cell count at the moment of diagnosis. The group of individuals HIV-infected when using drugs included those infected during nonmedical drug injection of drugs and psychoactive substances.

Virus RNA was isolated from $200 \mu \mathrm{L}$ of blood plasma using RealBest DeltaMag kits according to the manufacturer's protocol (Vector-Best, Russia). Amplification was performed using a lyophilized ready-to-use Reverse Transcription Master Mix containing all the components for a single-tube reverse transcription and PCR (Vector-Best, Russia) and an in-house set of primers. This allowed for amplifying three HIV-1 specific fragments for each clinical sample, namely, two fragments of the pol gene encoding the protease-reverse transcriptase (PR-RT, $1400 \mathrm{nt}$ ), integrase (IN, $960 \mathrm{nt}$ ), and a fragment encoding a part of the major envelope protein, env (732 nt). Sequencing was conducted in a 3130xl (Applied Biosystems, United States) automated sequencer. All original sequence fragments of the pol and env gene regions were assembled in whole sequences in Sequencher 4.1 software (Gene Codes Corporation, Ann Arbor, MI, United States). The assembled sequences of pol fragments (PR-RT and IN) and $e n v$ gene fragments were compared to the corresponding reference sequences of various HIV-1 subtypes and recombinant forms extracted from the Los Alamos HIV-1 database using CLUSTALW Multiple Alignment and BioEdit software 7.2.5 [14]. Phylogenetic analysis was performed with MEGA 6.0.6 by neighbor-joining method with 1000 bootstrap replicates based on Kimura's two-parameter model [15]. Statistical significance of phylogenetic tree topologies was estimated using bootstrap analysis. New intersubtype or inter-CRF sequences were analyzed by recombination identification programs (RIP, https://www.hiv.lanl.gov/) and SimPlot 3.5.1 software using a $200 \mathrm{nt}$ window with tree construction by the neighbor-joining method applying Kimura's two-parameter substitution model. The possible intersubtype mosaicisms of URFs were screened using the online jpHMM program (http://jphmm.gobics.de/submission_hiv.html) [16]. The V3 loop sequences were analyzed to estimate the genotypic virus tropism and to confirm the phenotypic coreceptor specificity using the online tools Position-Specific Scoring Matrix (PSSM) (http://indra.mullins.microbiol.washington.edu/web -pssm/) and Geno2pheno [coreceptor] 2.5 (g2p) (http://coreceptor.bioinf.mpi-inf.mpg.de/index.php), additionally checking whether the sequence codes for positively charged amino acid residues at 11 and/or 25 codons of the V3 loop [17]. The analyzed pol gene sequences were assayed for the presence of mutations determining resistance to protease, reverse transcriptase, and integrase inhibitors (DR mutations) with the specialized online service (http://sierra2.stanford.edu/ sierra/). The transmitted DR mutations were determined based on the WHO-recommended list of mutations for DR surveillance [18].

The HIV-1 polymerase, integrase, and env sequence data were submitted to the GenBank under the accession numbers KX640117-KX640184, KX640185-KX640251, and KX640252-KX640297.

\section{Results}

3.1. Clinical Epidemiological Data on Patients. In order to assess the current molecular epidemic situation on HIV-1 spread, 74 individuals diagnosed with HIV were involved in this study. Table 1 lists the clinical epidemiological data for the examined patients. The HIV-specific fragments of necessary lengths were not obtained for two HIV-1 samples, so they were discarded. Correspondingly, the examined sample comprised 72 individuals, namely, 33 (45.8\%) men and $39(54.2 \%)$ women, with an average age of 35 years (range, 23-60); 33 persons reported injection drug use (IDU); five of them were sex workers; 41 individuals (56.9\%) were infected via heterosexual contacts; three of them were IDUs; and one 


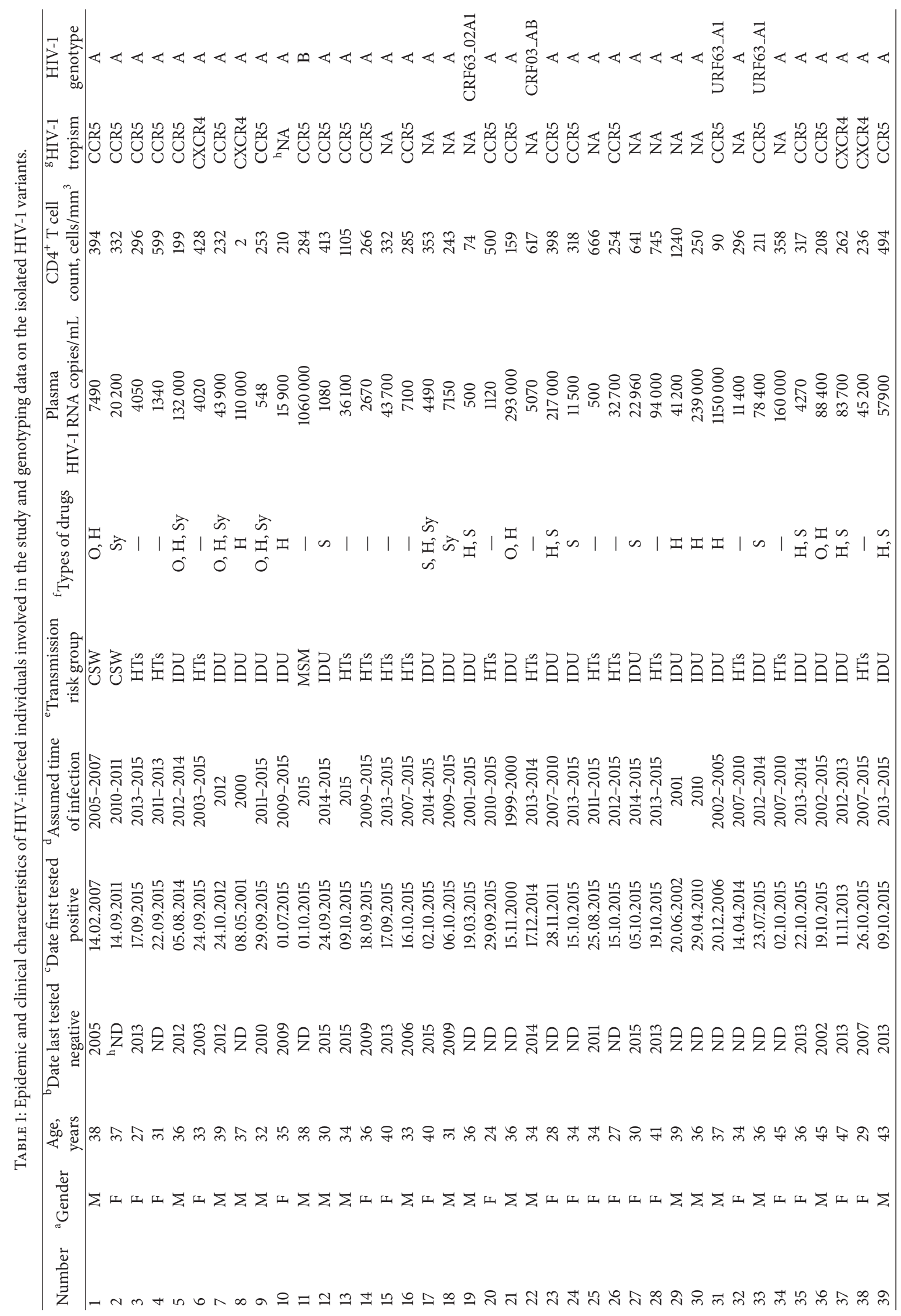




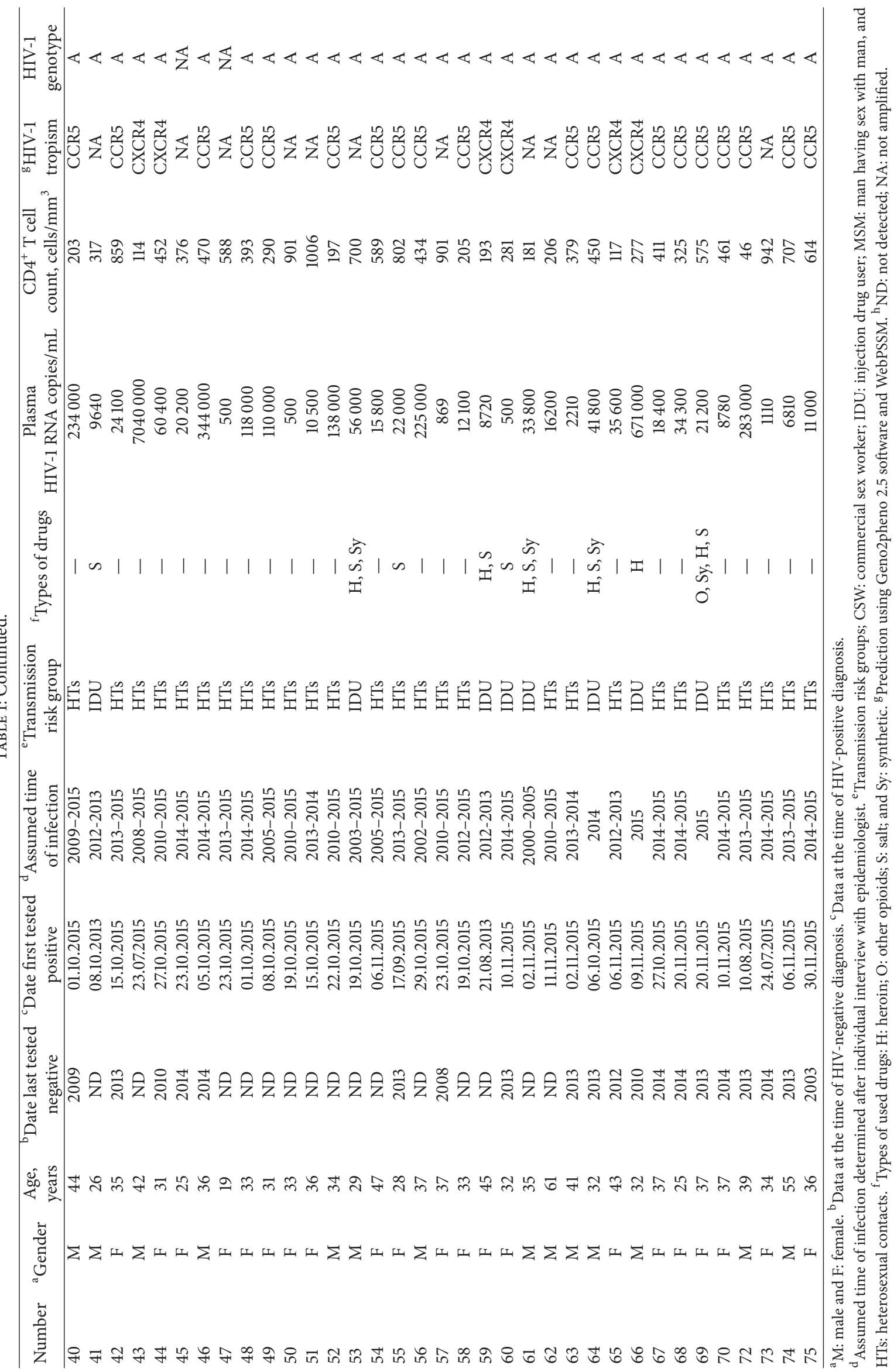


man was an MSM (1.4\%). Among the IDUs, $27.3 \%$ admitted intravenous use of heroin and/or fresh opium; $42.2 \%$ used salts and/or synthetic psychostimulants along with heroin/fresh opium; $27.3 \%$ used synthetic psychostimulants; and one participant refused to answer this question. Of them, $82.1 \%$ were residents of the city of Tyumen and $18.1 \%$ lived in other areas of the oblast. One person (no. 23) was putatively infected beyond the Tyumen Oblast and the remaining participants were infected in Tyumen/Tyumen Oblast. Putative time of infection was reliably enough detectable for $58.3 \%$ of the participants, since they had been earlier examined for HIV. In particular, $13.9 \%$ of the participants had been infected before 2010 and 44.4\%, presumably in 2010-2015. According to analysis of the clinical data, the median viral load and $\mathrm{CD}^{+}$cell counts were $2.2 \times 10^{4}\left(5.0 \times 10^{2}-7.0\right.$ $\left.\times 10^{6}\right)$ copies $/ \mathrm{mL}$ and $332(2-1240)$ cells $/ \mu \mathrm{L}$ for the overall sample and $2.2 \times 10^{4}\left(5.0 \times 10^{2}-3.4 \times 10^{5}\right)$ copies $/ \mathrm{mL}$ and 450 (46-1105) cells/ $\mu \mathrm{L}$ for the individuals infected in 20132015, respectively. Analysis of epidemiological data of the patients involved in the study demonstrated that this sample contained the clinical specimens of the individuals infected at different periods of the epidemic, including at least $44 \%$ of the specimens of the persons infected with HIV-1 after 2010. Examination of the genetic characteristics of HIV-1 variants isolated from the collected clinical specimens allows for molecular genetic description of the specific features in HIV infection epidemic and of the virus variants that determine the current epidemic in TO.

3.2. Analysis of Genetic Heterogeneity of the Virus Variants Isolated from HIV-Infected Individuals. The virus DNA was isolated from the blood plasma samples of 72 individuals, and the fragments encoding HIV-1 PR-RT (70 samples), IN (68 samples), and envelope protein (50 samples) were amplified and subject to phylogenetic analysis individually for each genome region. The phylogenetic trees constructed for the pol gene nucleotide sequences (PR-RT and IN) are shown in Figures 3 and 4. The genotyping of virus variants according to the PR-RT region demonstrates that one HIV1 variant (Tyumen 11) clustered with the HIV-1 subtype B; one (Tyumen 22) with CRF03_AB; and two (Tyumen 19 and Tyumen 33) with CRF63_02A1; the remaining assayed virus variants clustered with HIV-1 subtype A (A1). The following distribution of the analyzed virus specimens was observed for the HIV-1 IN region (Figure 4): Tyumen 11 clustered with subtype B; Tyumen 31 belongs to subtype A according to PR-RT and is intermediate between subtype A and CRF63 $02 \mathrm{~A} 1$ according to IN region, while Tyumen 33 (genotyped as CRF63 02A1 according to PR-RT) together with most HIV-1 variants clustered with subtype A.

The analysis of env gene region confirmed the Tyumen 11 clustering with subtype B, while Tyumen 31 and Tyumen 33 were genotyped as HIV-1 subtype A (Figure 5).

The conducted phylogenetic analysis revealed several epidemic networks in HIV-1 distribution. In particular, six pairs of genetically related HIV-1 variants were discovered; these variants clustered together in the phylogenetic tree constructed based on HIV-1 PR-RT sequences with bootstrap values of $\geq 99 \%$. Interestingly, these pairs unite the virus variants isolated from the individuals infected in different years (Tyumen 13 and Tyumen 21 and Tyumen 31 and Tyumen 58). For three of them, the branch topologies are also confirmed by the phylogenetic analysis based on the IN and/or env sequences (Tyumen 17-Tyumen 67, Tyumen 13Tyumen 21, and Tyumen 16-Tyumen 48), thereby suggesting a high probability of the epidemic relation between these pairs of patients.

As for the pair Tyumen 18-Tyumen 42 and Tyumen 23Tyumen 39, their support for sharing the same subbranch according to IN region was statistically insignificant (bootstrap values of $<70 \%$ ), while Tyumen 31 and Tyumen 58 variants belong to different HIV-1 genovariants (Figures 3 and 4 ). The obtained data suggest reinfection of the patients Tyumen 31, Tyumen 18, and/or Tyumen 42 and Tyumen 23 and/or Tyumen 39.

Summing up the genotyping results, we conclude that HIV-1 subtype A still remains predominant and determines development of the current epidemic in TO, its prevalence being 93.1\%. HIV-1 subtype B continues its circulation in MSM risk group and was detected in one case $(1.4 \%)$. The only cases of HIV-1 recombinant forms CRF63_02A1 (1.4\%) and CRF03_AB (1.4\%) were detected as well as two instances (Tyumen 31 and Tyumen 33) of HIV-1 unique recombinant forms, URF63_A1. The URF genome is mosaic, partially identical to subtype A and partially to CRF63_02A1, as was confirmed by phylogenetic analysis: some studied loci of the same isolate belong to subtype Al and the others to subtype 63_02A1 (Figures 3 and 4).

3.3. Analysis of Primary Resistance and Tropism of the Studied HIV-1 Variants. All HIV-1 nucleotide sequences encoding protease $(n=70)$, reverse transcriptase $(n=70)$, and integrase $(n=68)$ of the isolated virus variants were assayed for the presence of mutations influencing the resistance to the corresponding virus reproduction inhibitors, namely, the inhibitors of protease (PIs), reverse transcriptase (nonnucleoside and nucleoside inhibitors, NNRTIs, and NRTIs, resp.), and integrase (IIs) (Table 2).

The conducted analysis demonstrated a low prevalence of the primary drug resistance (DR) among the studied variants of HIV-1. Nonpolymorphic mutations determining HIV-1 resistance to NNRTIs, G190E, and Y181C were detected only in two HIV-1 specimens circulating in TO. The former mutation causes a high-level resistance to NVP, EFV, RPV, and ETR, while the latter is associated with a decreased response to the therapy with EFV. Likewise, two cases of the resistance to IIs were found. The corresponding mutations are T66I and E157Q. The former is a major mutation selected in the individuals treated with EVG, decreasing 15-fold the sensitivity to the drug, and the latter is a secondary mutation, reducing fivefold the sensitivity to RAL and halving the sensitivity to EVG.

The polymorphic mutations associated with drug resistance had different prevalence. The mutations able to improve HIV-1 reproductive characteristics in the presence of PIs or decrease the PI sensitivity (K20R, T74S, L10V/I, and K20I) were most frequently recordable among the examined HIV-1 variants, mainly in the individuals infected in 2010-2015. 


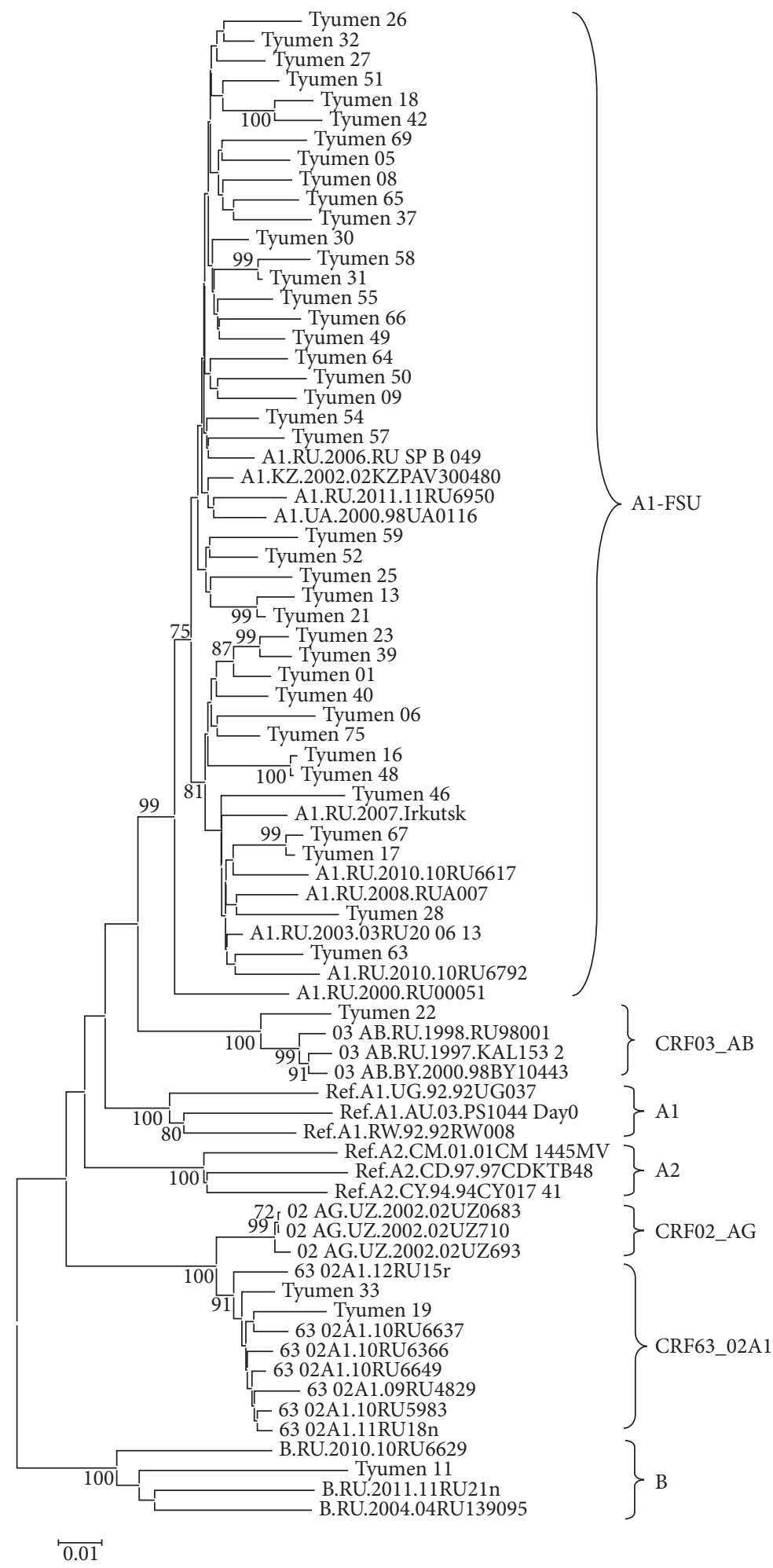

FIGURE 3: Neighbor-joining phylogenetic tree analysis of HIV-1 pol gene fragment (PR-RT) sequences from HIV-infected residents of Tyumen Oblast. Genetic distances were estimated using the Kimura's two-parameter model; clustering of strains was tested with 1000 bootstrap replicates; and statistical significance of the phylogenetic tree topology was estimated using bootstrap analysis. 


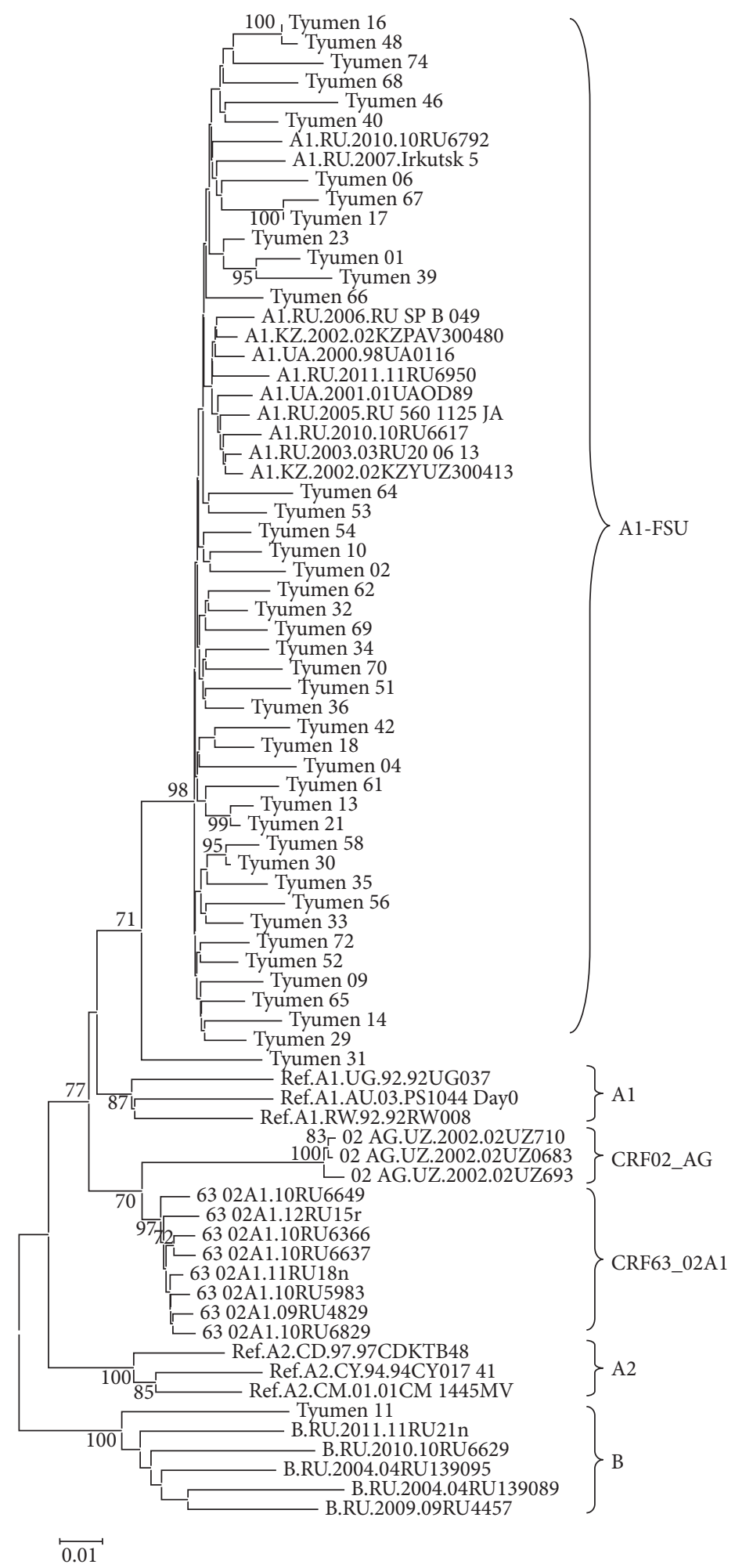

FIGURE 4: Neighbor-joining phylogenetic tree analysis of HIV-1 pol gene fragment (IN) sequences from HIV-infected residents of Tyumen Oblast. Genetic distances were estimated using Kimura's two-parameter model; clustering of strains was tested with 1000 bootstrap replicates; and statistical significance of the phylogenetic tree topology was estimated using bootstrap analysis. 


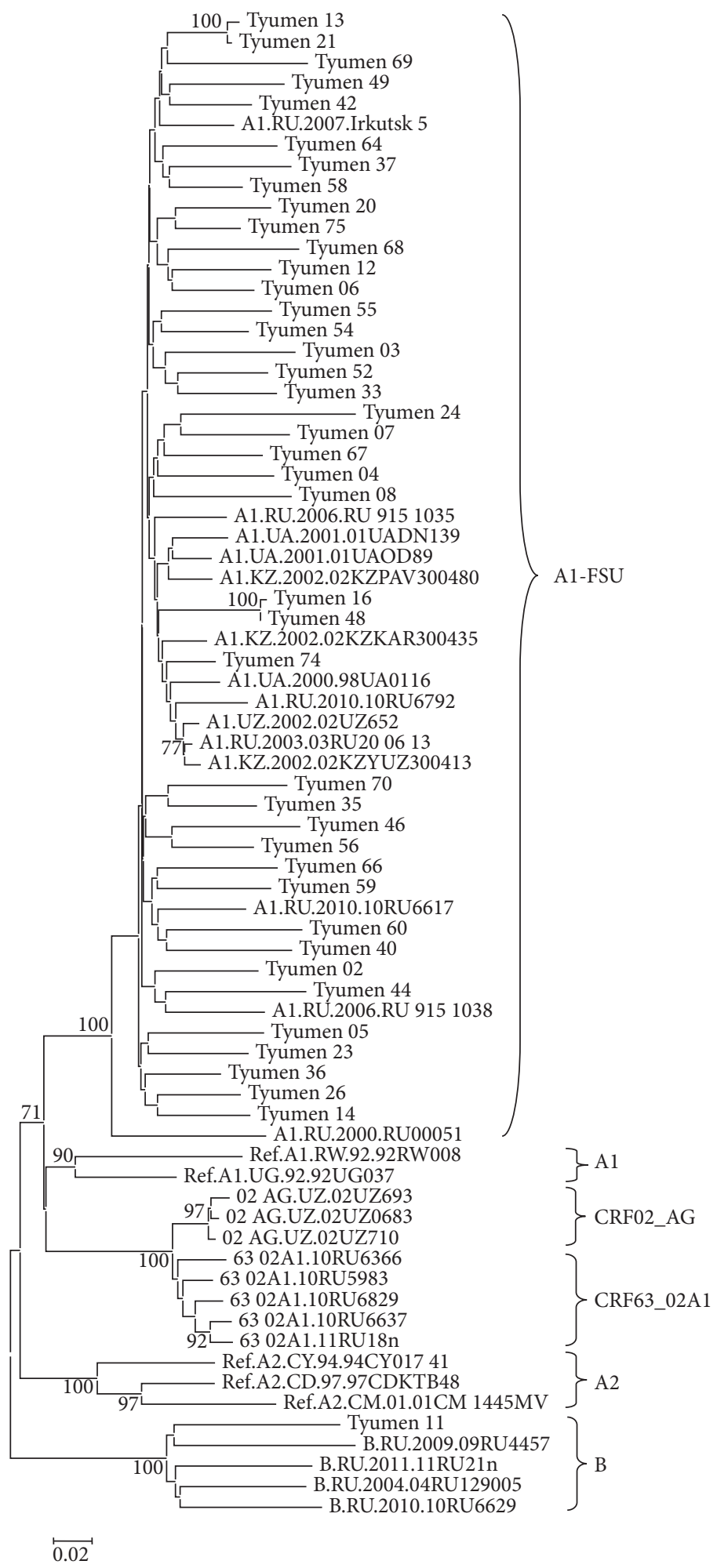

FIGURE 5: Neighbor-joining phylogenetic tree analysis of HIV-1 env gene fragment sequences from HIV-infected residents of Tyumen Oblast. Genetic distances were estimated using Kimura's two-parameter model; clustering of strains was tested with 1000 bootstrap replicates; and statistical significance of the phylogenetic tree topology was estimated using bootstrap analysis. 
TABLE 2: Detection rate and range of mutations in the pol gene in the examined HIV-1 variants that influence the sensitivity to virus reproduction inhibitors.

\begin{tabular}{lccc}
\hline & \multicolumn{2}{c}{ HIV-1 mutations associated with DR mutation (rate, \%) } \\
Resistance to PIs, $n=70^{*}$ & Resistance to NRTIs, $n=70^{*}$ & Resistance to NNRTIs, $n=70$ & Resistance to IIs, $n=68$ \\
\hline $\begin{array}{l}\text { K20R (12.5), T74S (12.5), L10V.I } \\
\text { (11.1), K20I (5.6), L33F (2.8), }\end{array}$ & A62V (8.3), V118I (6.9), T69S (4.2) & $\begin{array}{c}\text { E138A (4.2), G190E (1.4), Y181C } \\
\text { (1.4), N108I (1.4), V90I (1.4) }\end{array}$ & $\begin{array}{l}\text { L74I (94.1), T66I (1.5), T97A } \\
\text { (1.5), E157Q (1.5), T97A (1.5) }\end{array}$ \\
A71I (1.4) & & . &
\end{tabular}

${ }^{*} n$ : the number of analyzed sequences.

Solitary cases of the mutations influencing HIV resistance to PIs in combination with other DR mutations (L33F and A71I) were also recorded.

Of the mutations influencing NRTI therapy, only the V118I mutation (of the TAM set, efficient only in combination with other resistance mutations) was found. The mutations with a low effect on the resistance to NNRTIs (E138A, V108I, and V90I) also had a low prevalence. The sequences encoding HIV-1 IN carried the L74I in $94.1 \%$ of the examined subtype A viruses. This allows L74I to be regarded as a characteristic mutation for the HIV-1 subtype A population circulating in this area. On the other hand, only the A62VRT mutation of the DR mutations in the pol gene specific of HIV-1 subtype IDUA $\left(\mathrm{V}_{77 \mathrm{I}_{\mathrm{PR}}}\right.$ and $\mathrm{A} 62 \mathrm{~V}_{\mathrm{RT}}$ ) was detected at a rate of $8.3 \%$. The mutation $\mathrm{A} 62 \mathrm{~V}$ is an accessory mutation that often occurs in combination with the multinucleoside resistance mutations K65R or Q151M. Alone it does not reduce NRTI susceptibility.

The tropism of examined HIV-1 variants, that is, the use of CCR5/CXCR4 coreceptors, was predicted by analyzing the deduced amino acid sequences of HIV-1 env gene V3 loop. The prevalence of CXCR4-tropic variants among the examined HIV-1 specimens was $20 \%$; these variants were isolated from the individuals infected over 5 years ago in $25 \%$ of the cases versus $13.6 \%$ of the individuals infected less than 3 years ago.

\section{Discussion}

The circulation of resistant strains in the population of people living with HIV/AIDS (PLHIV) is a serious factor influencing the selection of first-line antivirals and adoption of the strategies for efficient ART schemes. Unlike the HIV IIs, which in this country are used only recently, the PIs and RTIs were and are still widely used for the specific ART. In Russia and the CIS countries, the HIV variants carrying DR mutations in pol gene are most frequently detectable in the individuals with inefficient ART. Among them, the mutations G190S, K103N, M184V, Y181C, K101E, M41L, and T215F/Y, influencing the HIV resistance to NRTIs and NNRTIs, are observed at a high rate [19-24]. Mutations K103N, M184I, T215Y, G190S, Y181C, $\mathrm{K} 65 \mathrm{R}$, and V108I with prevalences of 0.3 to $7 \%$ depending on the region are most frequently detected in the naïve HIV-infected individuals [21-28]. Only two HIV-1 variants carrying solitary G190E and Y181C mutations (causing resistance to RTI) and two HIV-1 specimens with resistance to IIs were found in the examined sample of HIV-1 specimens. Detection of the mutations associated with the resistance to IIs on the background of a very limited use of these drugs in clinical practice is explainable by a random selection of such substitutions. Despite a long-term practice of treatment with NRTIs, NNRTIs, and PIs, the transmission of resistant virus variants in the HIV population isolated from infected ART-naïve residents in TO was low. A low prevalence of the HIV-1 primary DR may be a favorable prognostic factor in the epidemic development in TO. However, note that over $10 \%$ of the HIV-1 variants circulating in TO had the mutations potentially associated with a decreased sensitivity to PIs and improvement of HIV reproductive characteristics in the presence of PIs.

The mutations $\mathrm{A} 62 \mathrm{~V}_{\mathrm{RT}}$ and $\mathrm{V} 77 \mathrm{I}_{\mathrm{PR}}$ are certain marker mutations for the subtype A HIV-1 variants circulating in Russia. The circulating subtype A HIV-1 variants characteristic of Russia are those carrying the $\mathrm{V} 77 \mathrm{I}_{\mathrm{PR}}$ and/or $\mathrm{A} 62 \mathrm{~V}_{\mathrm{RT}}$ mutations and wild-type (WT) HIV-1. The prevalence of such viruses among both the naïve HIV-infected individuals and the patients undergoing ARV therapy considerably varies ranging from solitary cases to $80-100 \%$ [2729]. Presumably, this is associated with the importation history of the different subtype A HIV-1 variants to certain regions of the country and with the specific features in subsequent progression of the epidemic. Wild-type HIV1 viruses were detected in TO as early as the late 1990s [30]. The observed low abundances of $\mathrm{A}^{2} 2 \mathrm{~V}_{\mathrm{RT}}$ (8.3\%) and $\mathrm{V} 77 \mathrm{I}_{\mathrm{PR}}(11.4 \%)$ among the HIV-1 variants in TO may result from importation of WT HIV-1 to this area followed by prevalent development of the "own" focus of HIV-infection epidemic.

The use of CCR 5 antagonists in the modern therapeutic schemes makes it necessary to study the tropism of the HIV1 variants isolated from the ART-naïve patients in order to assess the potential of such drugs [31-33]. According to several studies, the onset of HIV-1 infection with the spread of CCR5-tropic viruses in the body is characteristic of HIV1 subtype A as well as most virus genetic variants except for subtype D. Different studies on the use of CCR5 or CXCR4 coreceptors by HIV-1 belonging to non-B subtypes (including subtype A) involving naïve $\mathrm{HIV}^{+}$patients demonstrate that the abundance of CXCR4-tropic viruses varies from 2 to $40 \%$. This high variability may be influenced by different compositions of the studied populations and by differences in methodology. According to the data of most researchers, the detection rate of CXCR4-tropic HIV-1 variants in the patients at early disease state amounts to only several percent. Typically, the abundance of CCR5-tropic HIV variants decreases with the disease progression so that the CXCR4tropic viruses emerge only at later HIV infection stages [3443]. 
The results of our work demonstrate that the number of HIV-1 variants using CXCR4 coreceptor also increases with the duration of the disease among the studied subtype A viruses isolated from the patients with confirmed date of infection: the CXCR4-tropic viruses were isolated from $13.6 \%$ of the subjects infected several months to 3 years ago and $25 \%$ of the subjects infected over 5 years ago, which agrees with the data of other researchers. Nonetheless, the recorded levels of CXCR4-tropic HIV-1 variants among the patients infected for less than 3 years are rather high. Our results demonstrate that it is necessary to study the tropism of a virus before planning the use of CCR5 antagonists in the therapeutic schemes for the individuals infected with HIV-1 subtype A and to additionally study the prevalence of CXCR4-tropic HIV-1 variants among the naïve patients infected with other genetic variants specific of different regions of this country.

Comparison of the HIV-1 genetic variants isolated from the infected TO residents in the 2000s (2000-2009) and later (2010-2015) did not reveal any significant differences in the circulating virus variants. HIV-1 subtype A was and still remains the predominant variant. Phylogenetic analysis did not find any clustering of HIV-1 subtype A according to the time of infection and risk groups.

Different epidemic networks revealed by phylogenetic analysis of the HIV-1 variants, on the one hand, confirm that HIV infection spreads within local epidemic foci formed in TO during the previous years. On the other hand, the existence of such epidemic chains that include the HIV-1 variants with a high degree of genetic identity should be taken into account in a correct epidemiological investigation. Genetic identities of HIV-1 variants should be obligatorily assessed according to several genome regions. The revealed epidemic networks, which suggest that HIV is spread within an established focus of HIV infection, are also confirmed by analysis of the epidemic progression.

After 16 years of the epidemic in TO, the age cohort of 30-39 years has emerged to be maximally affected. The share of this age cohort is constantly growing, from $6.9 \%$ in 2000 to $48-50 \%$ in $2014-2015$. The share of the individuals over 50 years old is also constantly growing $(0.8 \%$ in 2000 to $4.6 \%$ in 2014). Presumably, part of the newly diagnosed patients has long been infected and, possibly, some "former" IDUs returned to using drugs and were thus involved into the epidemic. Epidemiological investigations recently performed when newly diagnosing a HIV infection demonstrates that HIV reinfection in the local foci of territorial epidemic remains rather high. The cause in the cohort free of drug addiction is a low sexual culture; as for the IDUs, especially using psychostimulants, they almost or completely neglect any of protection tools. Presumably, all of these factors also enhance HIV reinfection.

The detected HIV-1 URF63_A1, the so-called secondary recombinant forms, could have emerged only as a result of reinfection by different HIV-1 genetic variants (subtype $A$ and CRF63_02A1). In the examined group, one patient (Tyumen 31) infected by URF63_A1 practiced intravenous heroin use in the early 2000s and then had accidental unprotected sexual contacts and another patient (Tyumen 33) intravenously uses synthetic psychostimulants since 2012, having a drug-associated contact with a $\mathrm{HIV}^{+}$partner and accidental unprotected sexual contacts in his history. They have never left the TO area, suggesting that distinct HIV-1 URF63_A1 genetic variants circulate in TO. A case of isolation of an ancestral HIV-1 variant, CRF63_02A1, of a TO resident who is a long-term drug user confirms the possibility that new URFs emerge in TO. Presumably, the HIV-1 CRF63_02A1 originated from adjacent Siberian territories (Novosibirsk, Tomsk, and Kemerovo Oblasts; Figure 1), where this genetic variant is actively spreading in recent years [11-13].

Despite that the territorial epidemics in the Siberian region and TO display similar characteristics (distribution of $\mathrm{HIV}$-infected individuals according to infection routes, social parameters, gender, age, spread of drug abuse, and types of drugs), the epidemic in Siberia started with a delay of several years. Unlike Novosibirsk, Tomsk, and Kemerovo Oblasts, where the epidemic outbreaks with severalfold increase in the number of newly infected individuals were recorded in 2008-2014 (these territories are still at the top of the Russian rating for increase in newly infected persons), the last wave of HIV spread activation in TO was recorded in 2008-2011. Moreover, the epidemic growth rate was significantly lower: the maximal values recorded in 2009-2011 did not reach $20 \%$; moreover, a slow but stable trend of a decrease in the HIV spread is observed after 2011. Presumably, a likely reason underlying such a "controlled" epidemic in TO is its early onset and, as a consequence, timely development of the medical facilities able to constantly provide preventive activities, professional pretest and posttest services for HIVinfected individuals, and psychological consulting as well as maintenance of the patients from diagnosing and registration to observation and timely therapy. Note that the therapy coverage in TO in 2015 was $98 \%$ and its efficiency according to the approved criteria was $79.6 \%$ [44].

Despite an apparent positive dynamic of this epidemic, the number of HIV-infected individuals in TO continues to grow. Currently, TO is the region attractive for migrant workers from both the CIS countries and other Siberian regions, where the current epidemics are determined by HIV-1 recombinant forms, CRF02_AG and CRF63_02A1. Therefore, these HIV-1 genetic variants can be imported to TO and spread there, demonstrating the importance of monitoring of the spreading HIV-1 variants on a regular basis, especially, in remote areas with constant presence of seasonal migrant workers.

\section{Competing Interests}

The authors declare that there is no conflict of interests regarding the publication of this paper.

\section{Acknowledgments}

The study was supported by the Russian Foundation for Basic Research (Grant no. 16-15-10238). The authors thank all medical staff of the Tyumen Regional Center for Prevention and Control of AIDS and Infectious Diseases for their assistance in sampling. 


\section{References}

[1] Global AIDS Update, UNAIDS Report, 2016.

[2] News bulletin HIV-infection no. 40, http://www.hivrussia.org/ files/bul_40.pdf.

[3] A. Bobkov, R. Cheingsong-Popov, L. Selimova et al., "An HIV type 1 epidemic among injecting drug users in the former Soviet Union caused by a homogeneous subtype a strain," AIDS Research and Human Retroviruses, vol. 13, no. 14, pp. 1195-1201, 1997.

[4] A. A. Nabatov, O. N. Kravchenko, M. G. Lyulchuk, A. M. Shcherbinskaya, and V. V. Lukashov, "Simultaneous introduction of HIV type 1 subtype A and B viruses into injecting drug users in southern Ukraine at the beginning of the epidemic in the former Soviet Union," AIDS Research and Human Retroviruses, vol. 18, no. 12, pp. 891-895, 2002.

[5] A. Bobkov, E. Kazennova, T. Khanina et al., "An HIV type 1 subtype A strain of low genetic diversity continues to spread among injecting drug users in Russia: study of the new local outbreaks in Moscow and Irkutsk," AIDS Research and Human Retroviruses, vol. 17, no. 3, pp. 257-261, 2001.

[6] A. F. Bobkov, E. V. Kazennova, L. M. Selimova et al., "Temporal trends in the HIV-1 epidemic in Russia: predominance of subtype A," Journal of Medical Virology, vol. 74, no. 2, pp. 191196, 2004.

[7] E. V. Karamov, N. M. Gashnikova, I. G. Drozdov, and G. G. Onishchenko, Monitoring of HIV Infection in Eurasia. Atlas of the Human Immunodeficiency Virus, CERIS, Novosibirsk, Russia, 2009.

[8] M. M. Thomson, E. Vazquez de Parga, A. Vinogradova et al., "New insights into the origin of the HIV type 1 subtype A epidemic in former Soviet Union's countries derived from sequence analyses of preepidemically transmitted viruses," AIDS Research and Human Retroviruses, vol. 23, no. 12, pp. 1599-1604, 2007.

[9] United Nations Office on Drugs Crime (UNODC), The challenge of new psychoactive substances, Global Synthetics Monitoring: Analyses, Reporting and Trends (SMART) Programme, United Nations Office on Drugs Crime (UNODC), 2013, https://www.unodc.org/documents/scientific/NPS_2013_ SMART.pdf.

[10] Data from the UNODC Early Warning Advisory on New Psychoactive Substances, 2015, https://www.unodc.org/LSS/ Home/NPS.

[11] P. B. Baryshev, V. V. Bogachev, and N. M. Gashnikova, "Genetic characterization of an isolate of HIV type 1 AG recombinant form circulating in Siberia, Russia," Archives of Virology, vol. 157, no. 12, pp. 2335-2341, 2012.

[12] N. M. Gashnikova, V. V. Bogachev, P. B. Baryshev et al., "A rapid expansion of HIV-1 CRF63_02A1 among newly diagnosed HIV-infected individuals in the Tomsk Region, Russia," AIDS Research and Human Retroviruses, vol. 31, no. 4, pp. 456-460, 2015.

[13] N. M. Gashnikova, D. P. Zyryanova, E. M. Astakhova et al., "Predominance of CRF63_02A1 and multiple patterns of unique recombinant forms of CRF63_A1 among individuals with newly diagnosed HIV-1 infection in Kemerovo Oblast, Russia," Archives of Virology, 2016.

[14] T. A. Hall, "BioEdit: a user-friendly biological sequence alignment editor and analysis program for Windows 95/98/NT," Nucleic Acids Symposium Series, vol. 41, pp. 95-98, 1999.
[15] K. Tamura, G. Stecher, D. Peterson, A. Filipski, and S. Kumar, "MEGA 6: molecular evolutionary genetics analysis version 6.0," Molecular Biology and Evolution, vol. 30, no. 12, pp. 27252729, 2013.

[16] K. S. Lole, R. C. Bollinger, R. S. Paranjape et al., "Fulllength human immunodeficiency virus type 1 genomes from subtype C- infected seroconverters in India, with evidence of intersubtype recombination," Journal of Virology, vol. 73, no. 1, pp. 152-160, 1999.

[17] A. J. Low, L. C. Swenson, and P. R. Harrigan, "HIV coreceptor phenotyping in the clinical setting," AIDS Reviews, vol. 10, no. 3, pp. 143-151, 2008.

[18] D. E. Bennett, R. J. Camacho, D. Otelea et al., "Drug resistance mutations for surveillance of transmitted HIV-1 drugresistance: 2009 update," PLoS ONE, vol. 4, no. 3, Article ID e4724, 2009.

[19] A. N. Kolomeets, V. Varghese, P. Lemey, M. R. Bobkova, and R. W. Shafer, "A uniquely prevalent nonnucleoside reverse transcriptase inhibitor resistance mutation in Russian subtype A HIV-1 viruses," AIDS, vol. 28, no. 17, pp. F1-F8, 2014.

[20] A. N. Kolomeets, G. A. Kalacheva, L. I. Levakhina et al., "Specific epidemiological and molecular genetic features of the HIV infected individuals in the Siberian Federal District," MediAl, no. 1, pp. 16-20, 2012.

[21] O. A. Rumyantseva, I. A. Olkhovskiy, M. A. Malysheva et al., "Epidemiological networks and drug resistance of HIV type 1 in Krasnoyarsk region, Russia," AIDS Research and Human Retroviruses, vol. 25, no. 9, pp. 931-936, 2009.

[22] I. Lapovok, E. Kazennova, V. Laga et al., "Short communication: molecular epidemiology of HIV type 1 infection in Kazakhstan: CRF02-AG prevalence is increasing in the southeastern provinces," AIDS Research and Human Retroviruses, vol. 30, no. 8, pp. 769-774, 2014.

[23] V. Laga, I. Lapovok, E. Kazennova et al., "The genetic variability of HIV-1 in Kyrgyzstan: the spread of CRF02_AG and subtype A1 recombinants," Journal of HIV and AIDS, vol. 1, no. 2, 2015.

[24] E. Kazennova, V. Laga, I. Lapovok et al., "HIV-1 genetic variants in the Russian far east," AIDS Research and Human Retroviruses, vol. 30, no. 8, pp. 742-752, 2014.

[25] A. L. Sukhanova, E. V. Bogoslovskaia, A. I. Kruglova et al., "Protease and reverse transcriptase genetic polymorphism in HIV type 1 subtype A variants predominating in CIS countries," Molecular Biology, vol. 39, pp. 1063-1071, 2005.

[26] E. V. Kazennova, I. A. Lapovok, V. Ye. Laga, A. V. Vasilyev, and M. R. Bobkova, "Natural polymorphisms of HIV-1 IDUA variant pol gene HIV," HIV Infection and Immunosuppressive Disorders, vol. 4, no. 4, pp. 44-51, 2012.

[27] E. Vázquez De Parga, A. Rakhmanova, L. Pérez-Álvarez et al., "Analysis of drug resistance-associated mutations in treatmentnaïve individuals infected with different genetic forms of HIV-1 circulating in countries of the former Soviet Union," Journal of Medical Virology, vol. 77, no. 3, pp. 337-344, 2005.

[28] A. Vinogradova, E. Gafurova, M. Muñoz-Nieto, A. Rakhmanova, S. Osmanov, and M. M. Thomson, "Short communication: molecular epidemiology of HIV type 1 in the republic of dagestan, Russian federation: virtually uniform circulation of subtype A, former soviet union variant, with predominance of the V77IPR subvariant," AIDS Research and Human Retroviruses, vol. 26, no. 4, pp. 395-400, 2010.

[29] N. M. Gashnikova, A. V. Totmenin, S. R. Sauhat et al., "Molecular epidemiological characteristics of the HIV 1 transmission 
in Southern Russia," HIV Infection and Immunosuppressive Disorderse, vol. 5, no. 4, pp. 73-81, 2013.

[30] N. I. Roudinskii, A. L. Sukhanova, E. V. Kazennova et al., "Diversity of human immunodeficiency virus type 1 subtype A and CRF03_AB protease in Eastern Europe: Selection of the V77I variant and its rapid spread in injecting drug user populations," Journal of Virology, vol. 78, no. 20, pp. 11276-11287, 2004.

[31] Z. L. Brumme, J. Goodrich, H. B. Mayer et al., "Molecular and clinical epidemiology of CXCR4-using HIV-1 in a large population of antiretroviral-naive individuals," Journal of Infectious Diseases, vol. 192, no. 3, pp. 466-474, 2005.

[32] W. Huang, S. H. Eshleman, J. Toma et al., "Coreceptor tropism in human immunodeficiency virus type 1 subtype D: high prevalence of CXCR4 tropism and heterogeneous composition of viral populations," Journal of Virology, vol. 81, no. 15, pp. 78857893, 2007.

[33] M. C. A. Sucupira, S. Sanabani, R. M. Cortes et al., "Faster HIV-1 disease progression among Brazilian individuals recently infected with CXCR4-utilizing strains," PLoS ONE, vol. 7, no. 1, Article ID e30292, 2012.

[34] W. Huang, J. Toma, E. Stawiski et al., "Characterization of human immunodeficiency virus type 1 populations containing CXCR4-using variants from recently infected individuals," AIDS Research and Human Retroviruses, vol. 25, no. 8, pp. 795802, 2009.

[35] K. Chalmet, K. Dauwe, L. Foquet et al., "Presence of CXCR4Using HIV-1 in patients with recently diagnosed infection: correlates and evidence for transmission," Journal of Infectious Diseases, vol. 205, no. 2, pp. 174-184, 2012.

[36] P. Rieder, B. Joos, A. U. Scherrer et al., "Characterization of human immunodeficiency virus type 1 (HIV-1) diversity and tropism in 145 patients with primary HIV-1 infection," Clinical Infectious Diseases, vol. 53, no. 12, pp. 1271-1279, 2011.

[37] Q. Ataher, S. Portsmouth, L. A. Napolitano et al., "The epidemiology and clinical correlates of HIV-1 co-receptor tropism in non-subtype B infections from India, Uganda and South Africa," Journal of the International AIDS Society, vol. 15, no. 1, article 2, 2012.

[38] B. Weiser, S. Philpott, T. Klimkait et al., "HIV-1 coreceptor usage and CXCR4-specific viral load predict clinical disease progression during combination antiretroviral therapy," AIDS, vol. 22, no. 4, pp. 469-479, 2008.

[39] J. Esbjörnsson, F. Månsson, W. Martínez-Arias et al., "Frequent CXCR4 tropism of HIV-1 subtype A and CRF02_AG during late-stage disease-indication of an evolving epidemic in West Africa," Retrovirology, vol. 7, article 23, 2010.

[40] J. M. Baeten, B. Chohan, L. Lavreys et al., "HIV-1 subtype $\mathrm{D}$ infection is associated with faster disease progression than subtype A in spite of similar plasma HIV-1 loads," Journal of Infectious Diseases, vol. 195, no. 8, pp. 1177-1180, 2007.

[41] S. Raymond, P. Delobel, M. Mavigner et al., "CXCR4-using viruses in plasma and peripheral blood mononuclear cells during primary HIV-1 infection and impact on disease progression," AIDS, vol. 24, no. 15, pp. 2305-2312, 2010.

[42] R. M. Gulick, J. Lalezari, J. Goodrich et al., "Maraviroc for previously treated patients with R5 HIV-1 infection," New England Journal of Medicine, vol. 359, no. 14, pp. 1429-1441, 2008.

[43] L. Vandekerckhove, C. Verhofstede, and D. Vogelaers, "Maraviroc: integration of a new antiretroviral drug class into clinical practice," Journal of Antimicrobial Chemotherapy, vol. 61, no. 6, pp. 1187-1190, 2008.

[44] "The national recommendations on follow-up monitoring and therapy of HIV-infected patients," in Supplement to Epidemiology and Infectious Diseases. Topical Issues, chapter 3, section 3.2.5.2, no. 6, 2015 . 


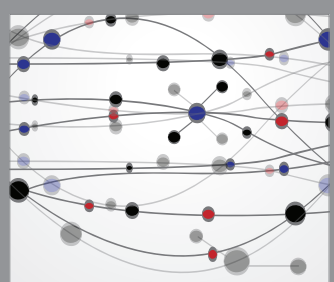

The Scientific World Journal
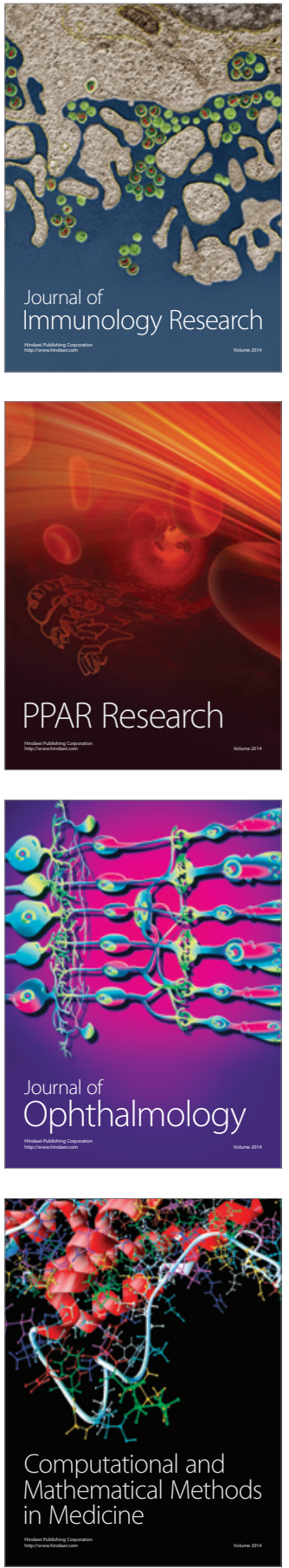

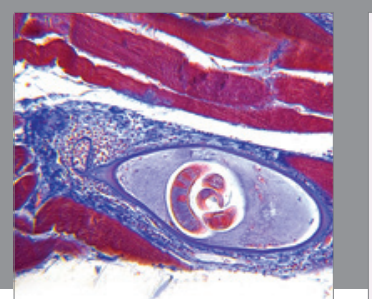

Gastroenterology Research and Practice

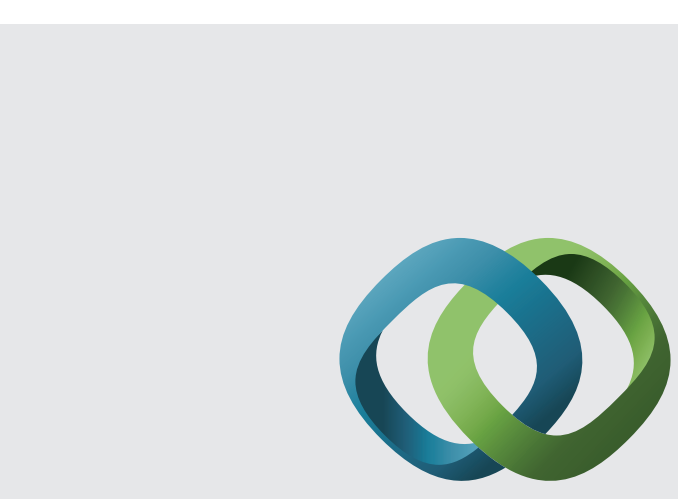

\section{Hindawi}

Submit your manuscripts at

http://www.hindawi.com
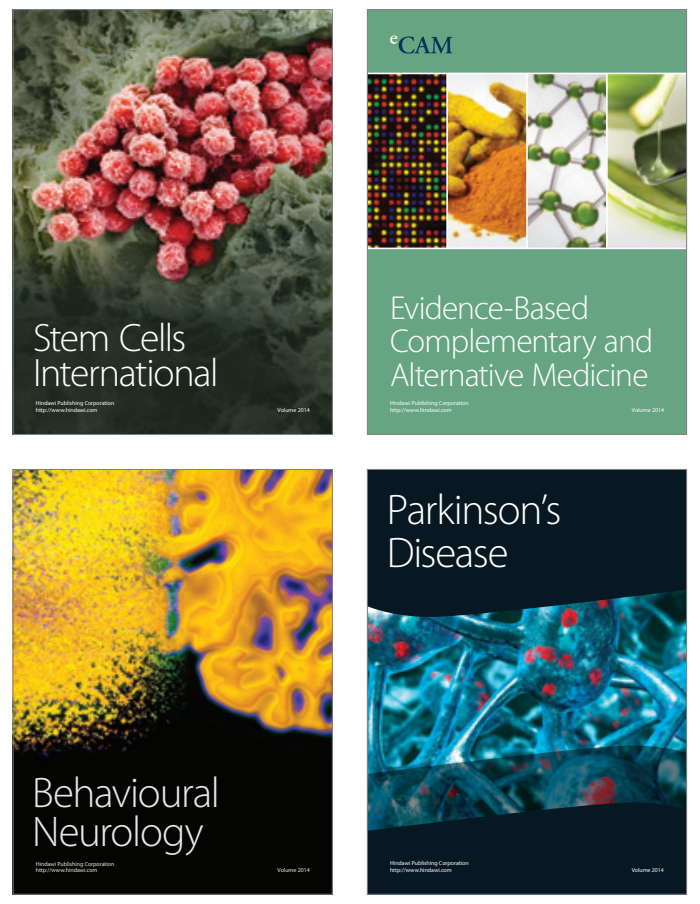
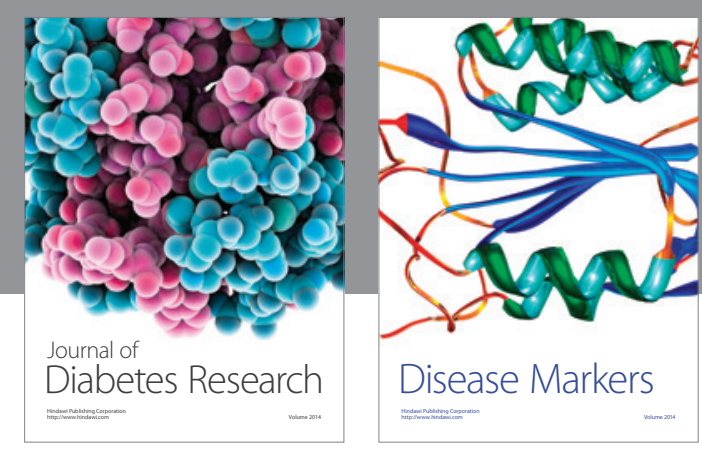

Disease Markers
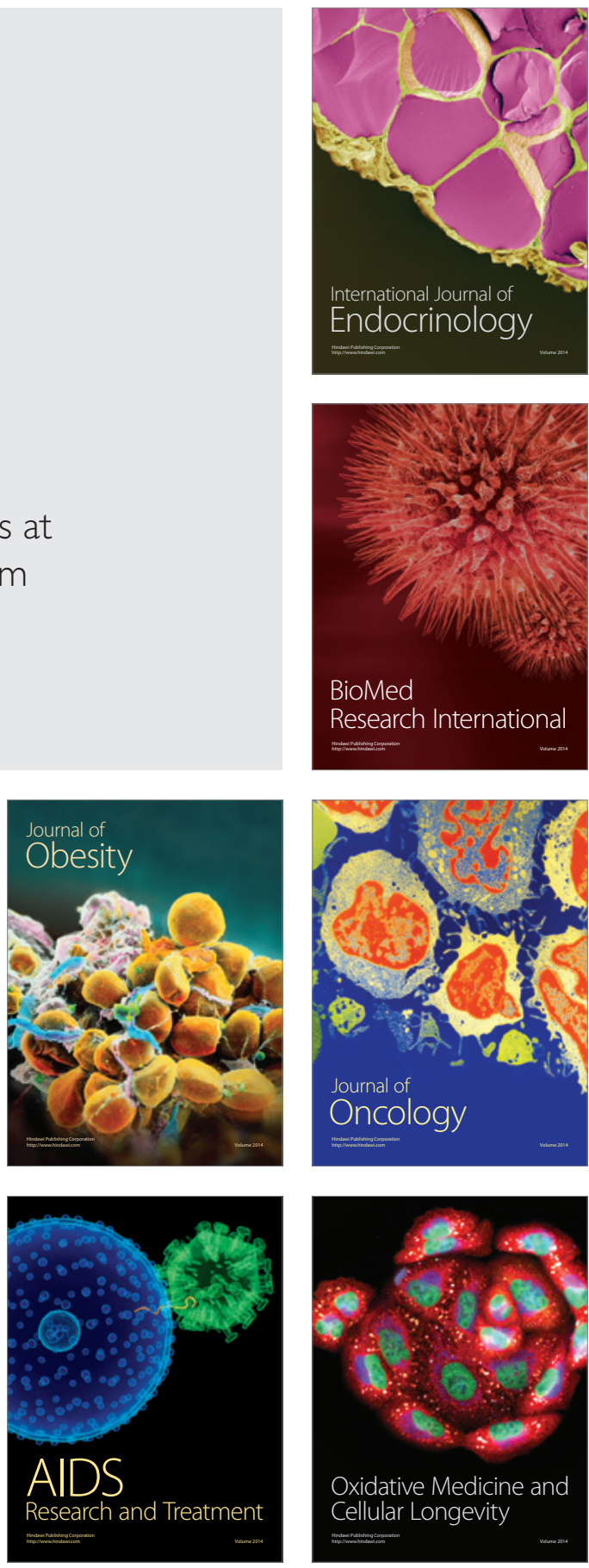